\title{
Muscle Imaging
}

\author{
William Palmer and M. K. Jesse
}

\section{Learning Objectives}

- Review the role of imaging in muscle diseases.

- Detect and classify acute muscle injury by imaging.

- Review unique imaging features in idiopathic inflammatory myopathy.

- Identify the imaging features of other common infectious, traumatic, and vascular muscle pathologies.

- Discuss the differential considerations in muscle lesions.

\subsection{Introduction to Muscle Imaging}

Evaluation and characterization of skeletal muscle pathology is a frequently encountered indication for musculoskeletal imaging. Causes of muscle pathology are diverse and include traumatic, autoimmune, infectious, inflammatory, neurologic, and neoplastic. Each etiology while dramatically different in the pathophysiology may present with similar imaging features. An understanding of the subtle differences in imaging features between the pathologic conditions may serve to guide diagnosis and treatment in these often complex cases. In this section, we will discuss the various skeletal muscle pathologies and the imaging features associated with each.

\subsection{Imaging Modalities in Skeletal Muscle Evaluation}

Radiograph, while excellent for bone pathology, has limited utility in the evaluation of muscle. Although the majority of muscle pathology is occult on routine radiographic images, $\mathrm{X}$-ray may be useful in a few conditions. Certain inflammatory or autoimmune myopathy, for example, is characterized by unique soft tissue calcifications of which radiographs may be the most reliable modality for detection. Magnetic resonance imaging and ultrasound offer excellent special resolution, allowing for the detailed evaluation of muscle microanatomy. Ultrasound offers an added benefit of dynamic imaging but is less sensitive than MRI for muscle edema and low-grade injury. Because of the superior sensitivity in detecting subtle injury, MR imaging evaluation is largely considered the diagnostic gold standard.

\subsection{Traumatic Muscle Injuries}

Muscle injury is common among athletes and poses a serious limitation to continued performance. The location and extent of a muscle injury has implications on the recovery and functional outcome.

\subsubsection{Muscle Strain/Tear}

\section{Key Point}

- Acute muscle strains/tears most frequently involve the gastrocnemius, biceps femoris, and rectus femoris muscles of the lower extremity.
Department of Radiology, Mass General Hospital,

Boston, MA, USA

e-mail: wpalmer@partners.org

M. K. Jesse

Department of Radiology, University of Colorado Hospital,

Aurora, CO, USA 
Acute muscle strains/tears are commonly encountered muscle injury in the athlete, most frequently involving the gastrocnemius, biceps femoris, and rectus femoris muscles of the lower extremity $[1,2]$. Muscle strains are characterized into three grades with progressing severity and complexity of the injury and increasing time to recovery (Table 17.1).

Table 17.1 Grades of muscle strain [3-6]

\begin{tabular}{|c|c|c|c|}
\hline Injury & MR findings & $\begin{array}{l}\text { Extent of } \\
\text { injury }\end{array}$ & Example \\
\hline Grade 1 & $\begin{array}{l}\text { T2 high signal edema with } \\
\text { feathered appearance, usually } \\
\text { at the myotendinous junction. } \\
\text { No architectural distortion } \\
\text { - Thickened or edematous } \\
\text { tendon without tear or laxity }\end{array}$ & $\begin{array}{l}<10 \% \text { fiber } \\
\text { disruption }\end{array}$ & $\begin{array}{l}\text { Figure } \\
17.1\end{array}$ \\
\hline Grade 2 & $\begin{array}{l}\text { - T2 high signal edema with } \\
\text { focal/partial fiber disruption } \\
\text { - } \pm \text { intramuscular hematoma }\end{array}$ & $\begin{array}{l}10-50 \% \\
\text { fiber } \\
\text { disruption }\end{array}$ & $\begin{array}{l}\text { Figure } \\
17.2\end{array}$ \\
\hline Grade 3 & $\begin{array}{l}\text { - Complete muscle fiber/tendon } \\
\text { disruption with laxity with/ } \\
\text { without retraction } \\
\text { - intramuscular hematoma }\end{array}$ & $\begin{array}{l}50-100 \% \\
\text { fiber } \\
\text { disruption }\end{array}$ & $\begin{array}{l}\text { Figure } \\
17.3\end{array}$ \\
\hline
\end{tabular}

\subsubsection{Delayed Onset Muscle Soreness}

Subacute or delayed muscle injury can be seen in the setting of overuse and is referred to in radiology literature as delayed onset muscle soreness (DOMS). DOMS is thought to occur as a result of muscle fiber microtrauma followed by a delayed inflammatory phase. This injury, while painful, results in no permanent muscle damage or functional deficit. Ultrasound interrogation often yields diffuse hyperechoic foci in an enlarged muscle belly but may be insensitive in mild injury [7]. Fluid-sensitive MR imaging is the gold standard diagnostic exam, demonstrating diffuse muscle edema involving all utilized muscles of a compartment [8] (Fig. 17.4).

\subsubsection{Muscle Herniation}

Muscle hernias, or myofascial defects, represent focal protrusion of the muscle fibers through a focal defect in the overlying superficial muscle fascia. Muscle hernias are most commonly seen in the lower extremity with the anterior compartment of the lower leg the most common location. Ultrasound is the
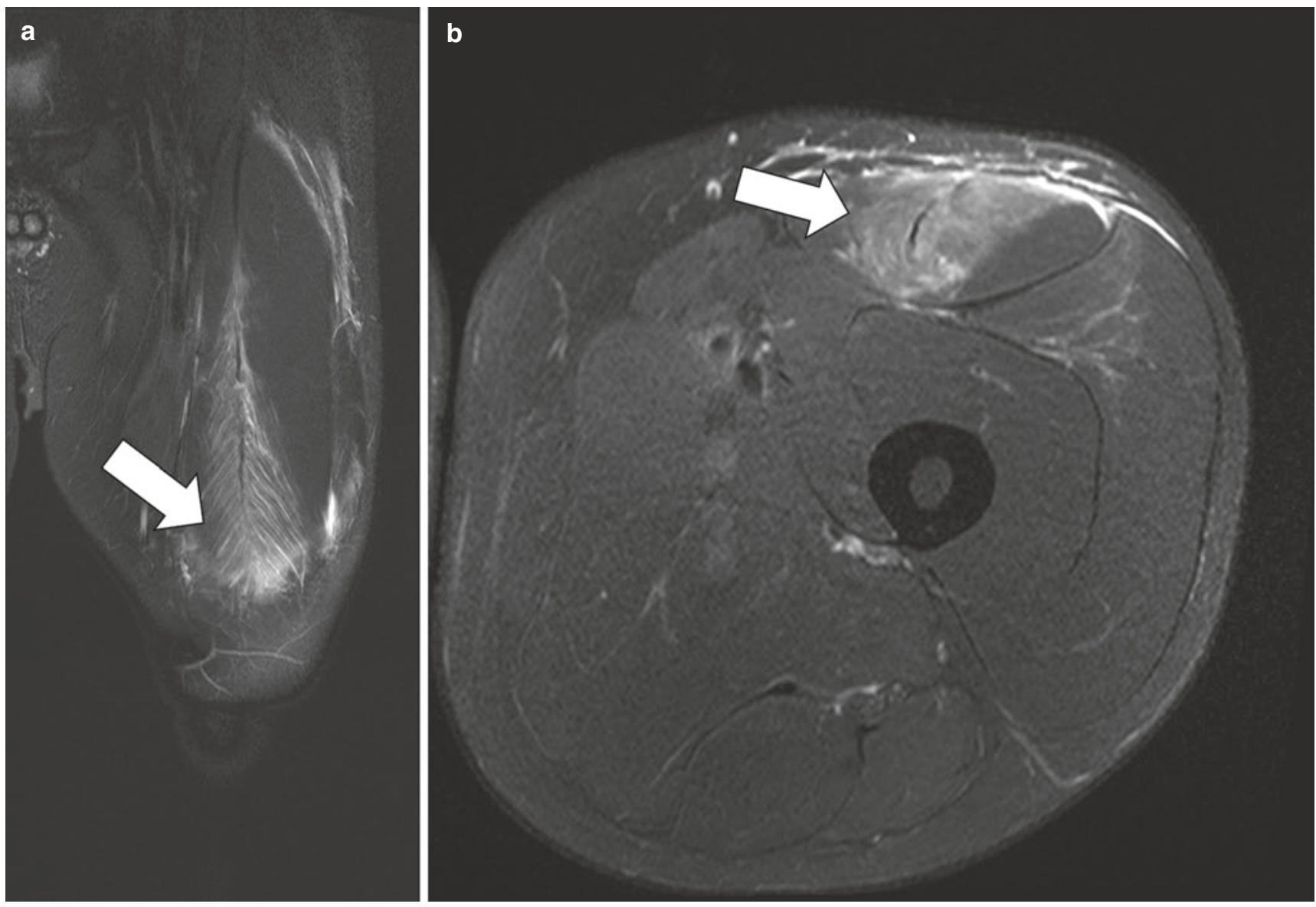

Fig. 17.1 Coronal (a) and axial (b) fat-saturated STIR MR images of the left thigh demonstrate feathered edema along the myotendinous junction of the rectus femoris muscle. No fiber disruption is identified in keeping with a grade 1 muscle injury 


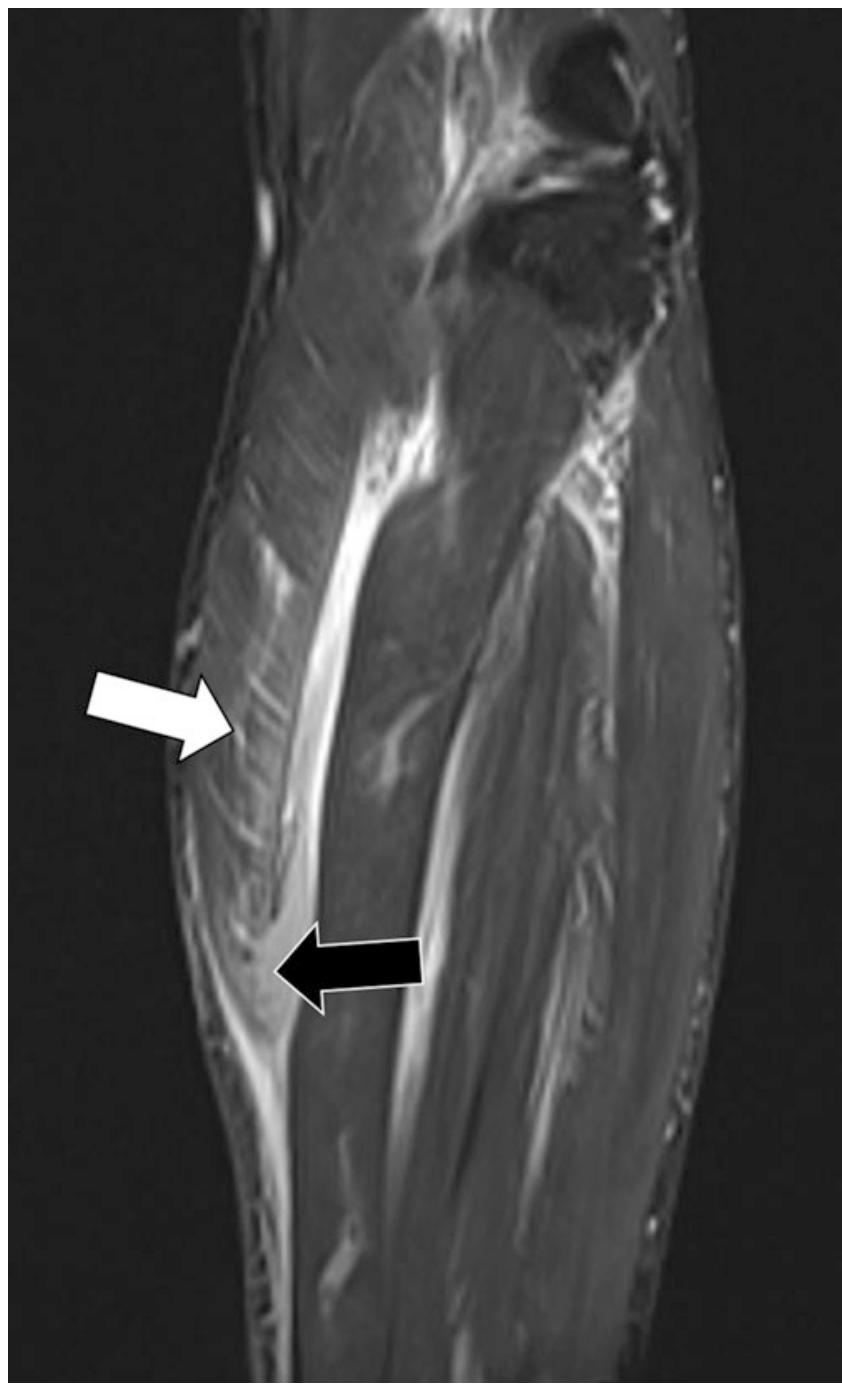

Fig. 17.2 Coronal fat-saturated STIR MR image of the lower leg demonstrates extensive intramuscular and fascial edema at the distal gastrocnemius muscle (white arrow). Partial-thickness fiber disruption at the distal myotendinous junction (black arrow) is in keeping with grade 2 muscle injury

modality of choice for the evaluation of muscle hernia as it may provide dynamic visualization of a reducible protrusion. Herniated muscle on US appears hypoechoic and mushroomshaped with a smaller neck at the location of the fascia [9] (Fig. 17.5). MRI may be useful in the quantification of the fascial defect and herniated muscle bulk [10].

\subsection{Inflammatory Myopathies}

Inflammatory myopathy is classified as idiopathic or secondary. Secondary inflammatory myopathy is most frequently associated with autoimmune/connective tissue disorders such as Sjogren's disease or systemic lupus but can also be seen as a consequence of endocrine disorders or paraneoplastic syndromes. Inflammatory myopathy is a subset of heterogeneous muscle diseases that share similar pathophysiology, imaging features, and clinical presentation. Progressive and symmetric proximal to distal muscle pain and weakness in the setting of elevated muscle enzymes and abnormal EMG is suggestive of idiopathic myositis. MRI is the gold standard modality for the identification and characterization of these disorders.

\subsubsection{Polymyositis/Dermatomyositis}

Polymositis and dermatomyositis are related idiopathic inflammatory diseases of muscle that are characterized by muscle pain, weakness, and edema in a proximal to distal progressive distribution often involving the anterior compartment musculature of the thigh [11]. On MRI, both entities are defined by muscle edema involving one or more muscles in a symmetric distribution [12]. These entities while similar differ slightly in clinical and imaging features. Dermatomyositis, for example, is often seen in conjunction with esophageal dysfunction and may present with classic "sheet-like" cutaneous/dermal calcifications [13] (Fig. 17.6). The microanatomy of muscle involvement between these two entities also differs, with the endomysium (lining between small muscle fibers) preferentially involved in polymyositis and the perimysium and epimysium (around the larger muscle fascicles and muscle belly) preferentially involved in dermatomyositis. While both entities are characterized by feathered muscle edema in the proximal muscle groups, the differences in preferential involvement may help to differentiate these two on MR imaging. Muscle edema in polymyositis is seen centrally within the muscle belly, often sparing the peripheral muscle fascia and myotendinous junction (Fig. 17.7). Dermatomyositis by contrast presents as muscle edema predominantly peripheral with notable myotendinous and myofascial involvement [14, 15] (Fig. 17.8).

\section{Key Point}

- Muscle edema in polymyositis is seen centrally within the muscle belly, often sparing the peripheral muscle fascia and myotendinous junction. 

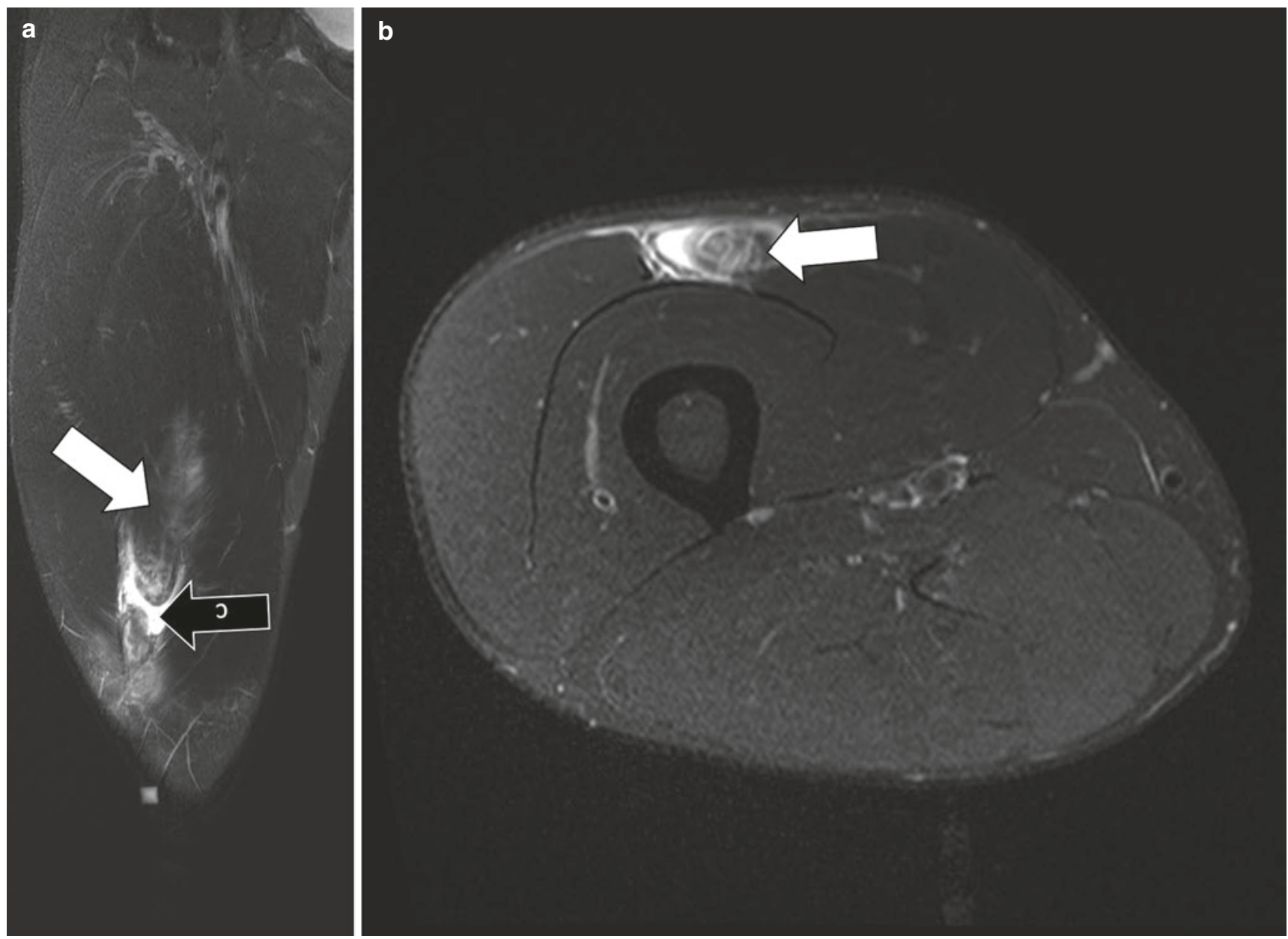

Fig. 17.3 Coronal (a) and axial (b) fat-saturated STIR MR images of the right thigh demonstrate extensive edema in the distal rectus femoris muscle belly (white arrows). This is a grade 3 injury given the complete fiber disruption and retraction apparent on the coronal image (black arrow)

\subsubsection{Inclusion Body Myositis}

Inclusion body myositis (IBM) is the most common idiopathic myopathy and is distinctly different in its pathophysiology compared to other idiopathic conditions. IBM is defined by inclusions of amyloid-B-protein within the skeletal muscle [16]. The onset of IBM is more common in the elderly and is often a distal to proximal distribution. MR imaging can easily differentiate IBM through the classic muscle involvement and the presence of often profound fatty infiltration [17, 18] (Fig. 17.9). The medial head of the gastrocnemius and flexor digitorum profundus muscles are commonly involved. IBM also frequently involves the anterior compartment muscles of the thigh, but unlike polymyositis and dermatomyositis, the rectus femoris muscle is notably spared in IBM [14] (Fig. 17.10 and Table 17.2).

\subsection{Myonecrosis}

Myonecrosis is the infarction of skeletal muscle. It has many etiologies such as trauma (e.g., crush injury), compartment syndrome, prolonged immobilization, poorly controlled diabetes, radiation treatment, and toxin (e.g., snake venom) [19]. On MRI, the infarcted muscle typically demonstrates nonspecific swelling and edema with heterogeneity that may suggest the presence of necrosis [20]. The feathery, striated pattern of muscle architecture usually remains visible. After contrast administration, infarcted muscle does not enhance (Fig. 17.11). It may not be feasible to inject contrast, however, if acute myonecrosis is complicated by rhabdomyolysis. Myonecrosis is sometimes incomplete. Enhancing linear and curvilinear foci indicate residual viable muscle tissue along vascular pedicles [21]. Once the healing process begins, contrast MRI can show 


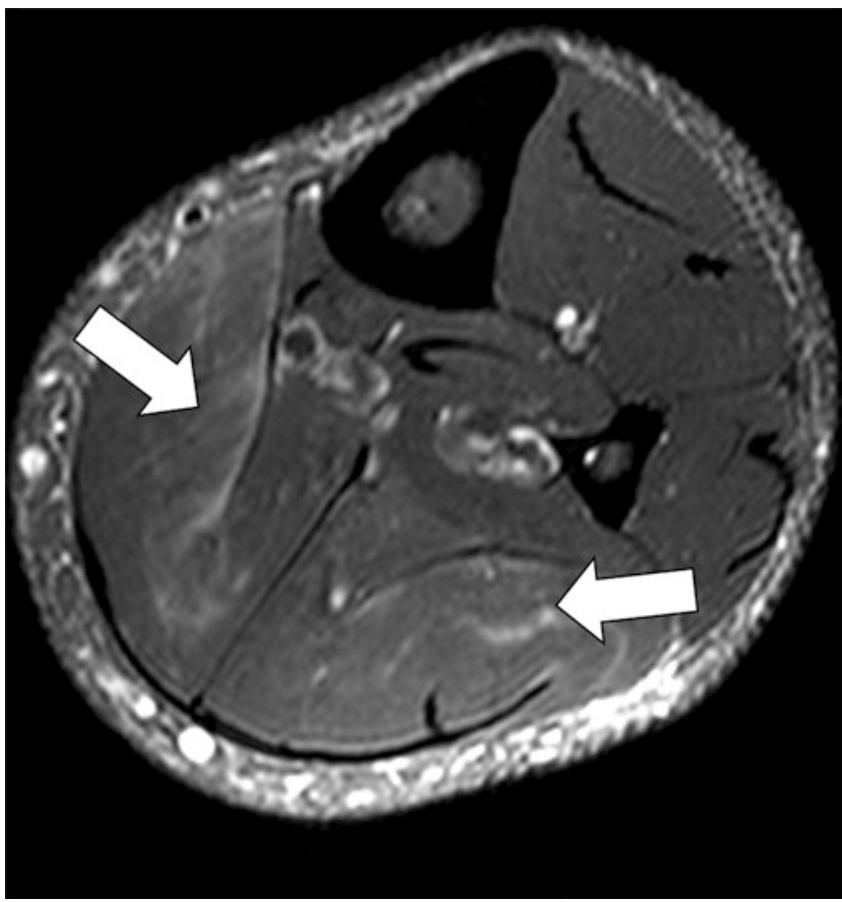

Fig. 17.4 Axial fat-saturated T2 MR image of the left calf in a 31-yearold male with severe calf pain after cross-fit workout. The feathered edema in the posterior compartment musculature (white arrows) is consistent with delayed onset muscle soreness (DOMS). This finding may persist for months following the initial insult

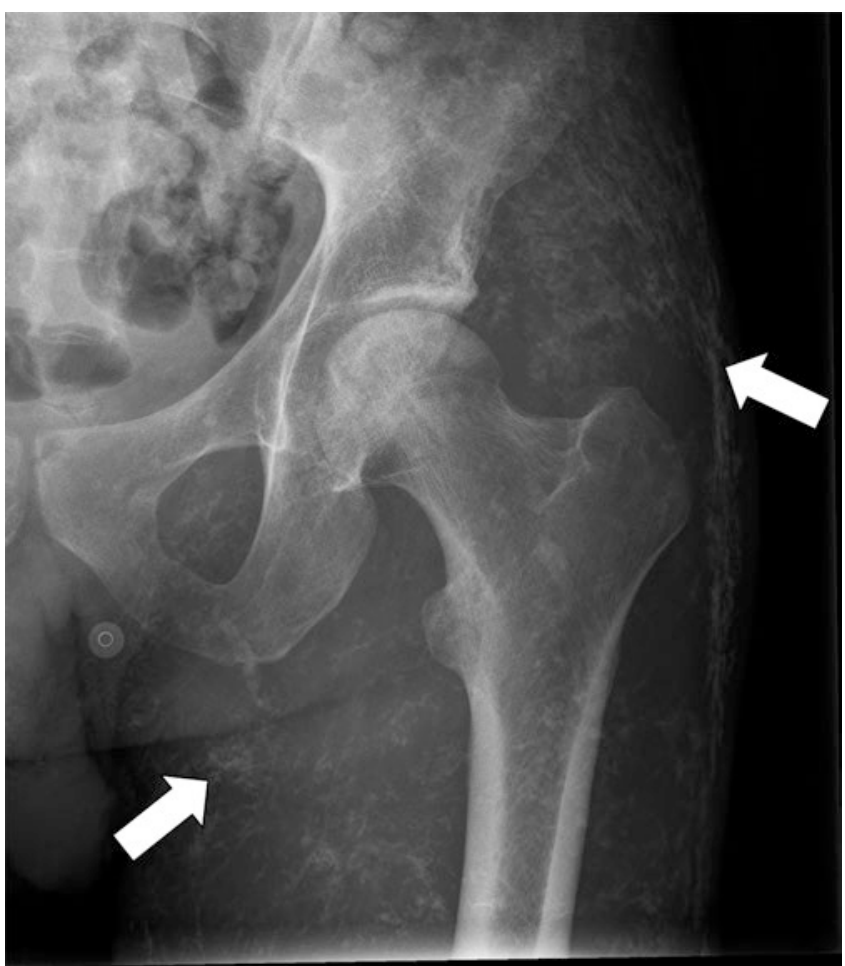

Fig. 17.6 Anteroposterior radiograph of the left hip demonstrates "sheet-like" linear calcifications (white arrows) within the soft issues in a patient with known dermatomyositis

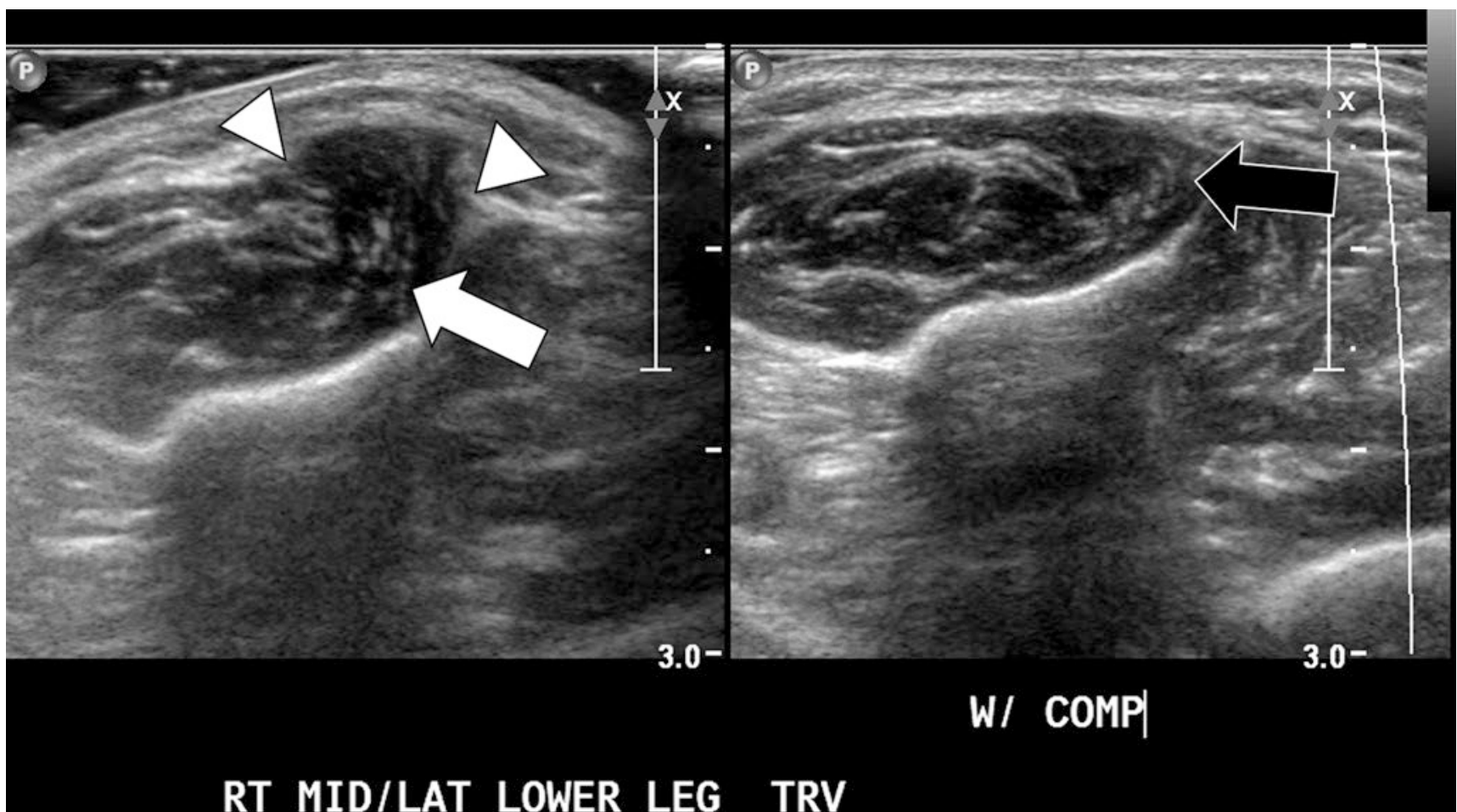

Fig. 17.5 Transverse gray-scale sonographic image of the right lower leg anterior compartment demonstrates a short segment defect in the superficial fascia (white arrowheads) with associated hypoechoic mushroom-shaped herniation of the peroneus longus muscle belly (white arrow). Comparison images of the normal left peroneus longus muscle are provided (black arrow) 
rim enhancement due to granulation tissue at the interface of viable and nonviable tissue. This appearance can be confusing because it can mimic a phlegmon or abscess. In the late stages of healing, chronic sequelae of myonecrosis include cystic cavitation and dystrophic calcification.

\subsection{Compartment Syndrome}

In compartment syndrome, elevated pressure reduces arterial blood flow to muscles. The clinical diagnosis is confirmed by

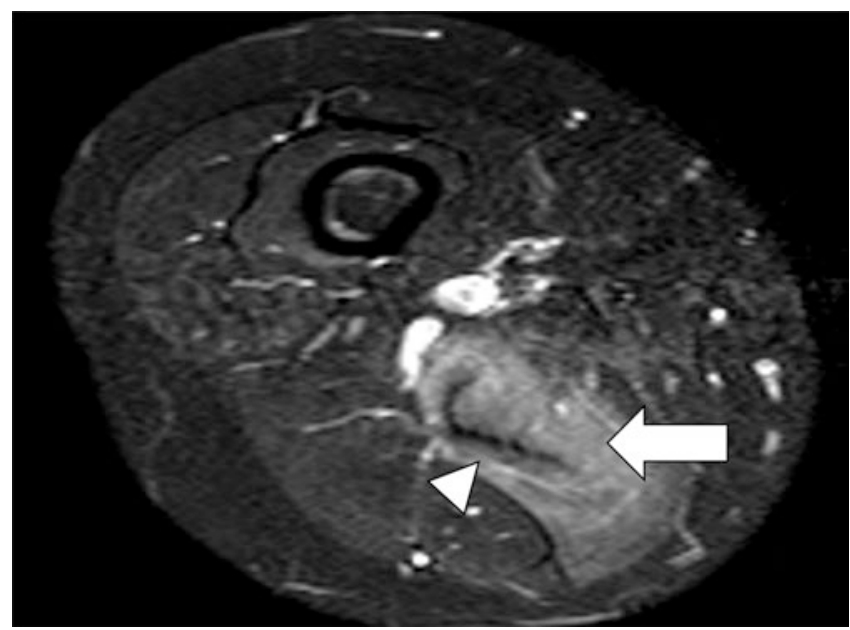

Fig. 17.7 Fat-saturated STIR MR image of the right thigh in a patient with known Sjogren's disease and polymyositis demonstrates marked diffuse edema isolated to the semimembranosus muscle (white arrow). Notice the diffuse endomysial pattern of muscle involvement with sparing of the fascia and myotendinous junction (white arrowhead)
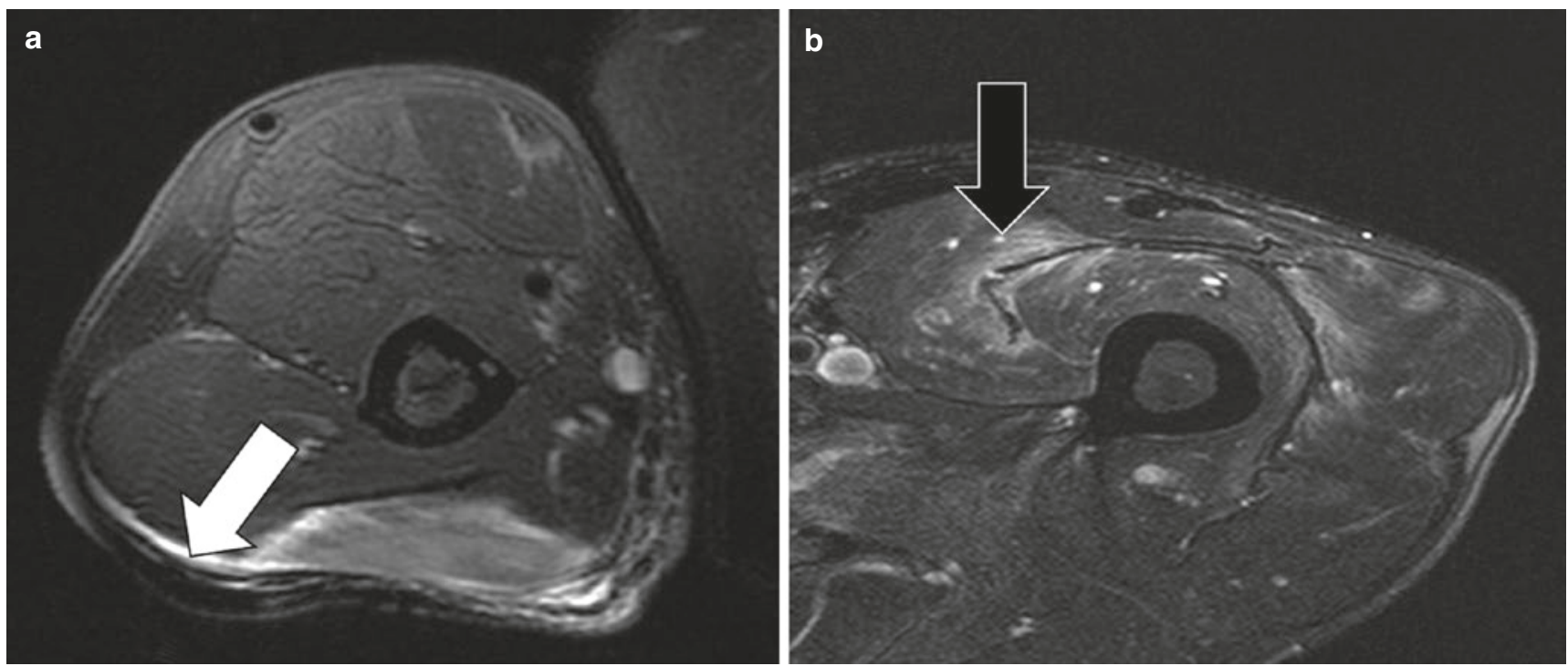

Fig. 17.8 Fat-saturated STIR MR images of the upper right arm (a) and right thigh (b) in a 27-year-old patient with dermatomyositis. Notice the perimysial pattern of edema with preferential involvement of the superficial fascia (white arrow) and myotendinous junction (black arrow) measuring intracompartmental pressure. Decreased circulation causes ischemia that can be reversed if the pressure is relieved. If intracompartmental pressure rises above perfusion pressure, blood flow is arrested causing irreversible myonecrosis. Infarcted muscle cannot be salvaged. The most common etiologies include trauma (crush injury, fracture), burn, overexertion, infection, and prolonged compression as might result from overly tight bandaging. The anterior compartment of the lower leg is particularly susceptible to compartment syndrome due to its confinement by surrounding

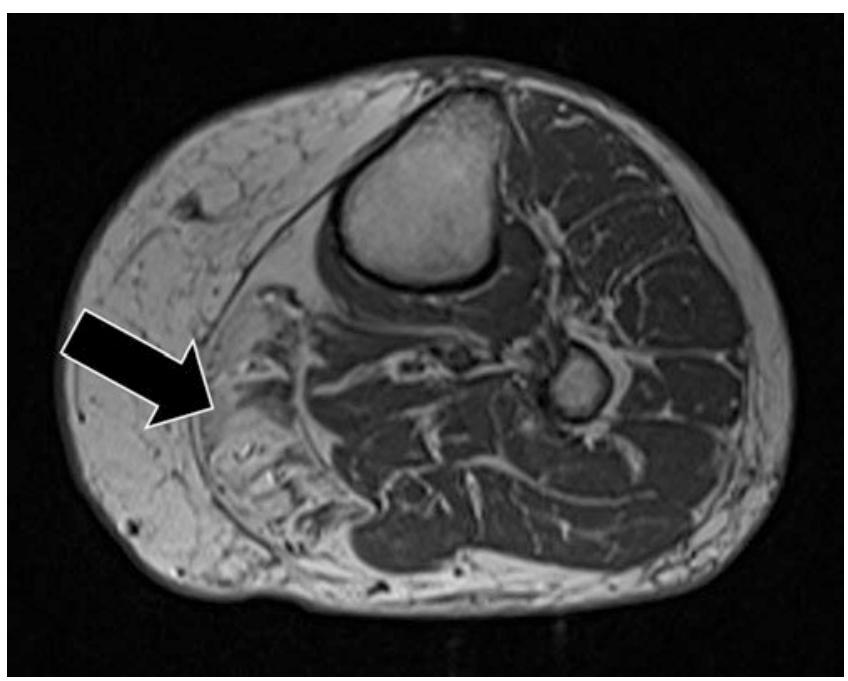

Fig. 17.9 Axial T1 MR image of the left lower leg in an 82-year-old male demonstrates profound isolated fatty infiltration of the medial head of the gastrocnemius muscle (black arrow). This pattern when symmetric is in keeping with chronic changes of inclusion body myositis 


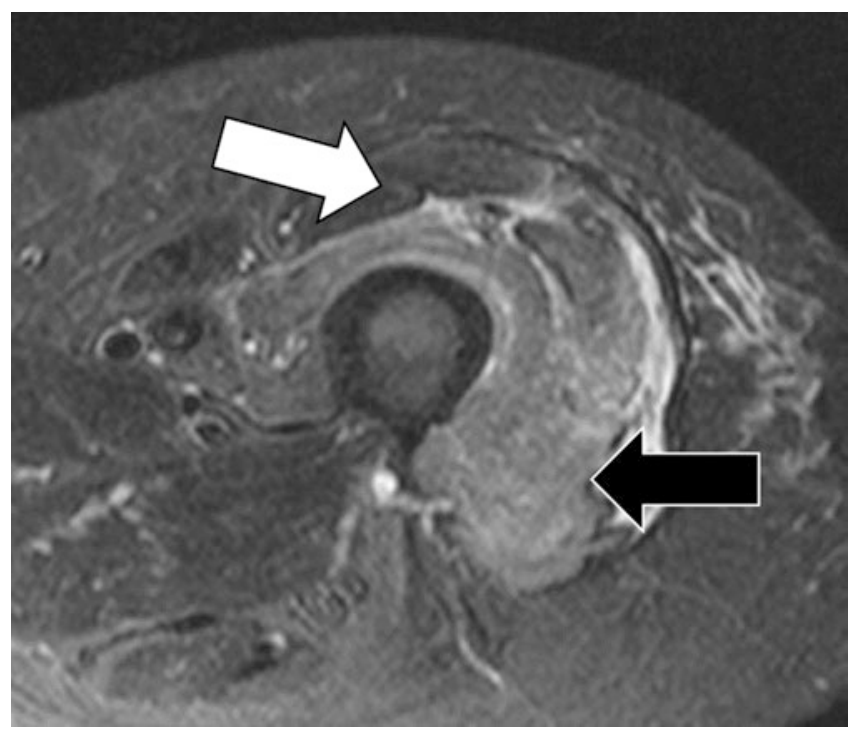

Fig. 17.10 Axial fat-saturated STIR MR image of the left thigh in a patient with inclusion body myositis and acute pain. Notice the diffuse edema in the anterior compartment musculature (black arrow) with notable sparing of the rectus femoris (white arrow)

Table 17.2 Clinical and imaging features of idiopathic myopathies $[11,12,14,17,18]$

\begin{tabular}{|c|c|c|}
\hline Polymyositis & Dermatomyositis & $\begin{array}{l}\text { Inclusion body } \\
\text { myositis }\end{array}$ \\
\hline $\begin{array}{l}\text { - Symmetric } \\
\text { - } \text { Proximal > distal } \\
\text { - Female > male } \\
\text { - Adults peak } 30-60 \\
\text { - Endomysial } \\
\text { involvement } \\
\text { (diffuse muscle } \\
\text { edema on MR) } \\
\text { - Anterior thigh } \\
\text { compartment }\end{array}$ & $\begin{array}{l}\text { - Symmetric } \\
\text { - Proximal > distal } \\
\text { - Female > male } \\
\text { - Juvenile and adult } \\
\text { onset } \\
\text { - Classic dermal } \\
\text { calcifications } \\
\text { - Perimysial } \\
\text { involvement (facial } \\
\text { or myotendinous } \\
\text { edema on MR) } \\
\text { - Anterior thigh } \\
\text { compartment }\end{array}$ & $\begin{array}{l}\text { - Symmetric } \\
\text { - Proximal = distal } \\
\text { - Male > female } \\
\text { - Elderly } \\
\text { predisposition } \\
\text { - Fatty infiltration } \\
\text { - Anterior } \\
\text { compartment with } \\
\text { rectus femoris } \\
\text { sparing } \\
\text { - Classic muscle } \\
\text { involvement } \\
\text { (medial head of the } \\
\text { gastrocnemius, } \\
\text { flexor digitorum } \\
\text { profundus) }\end{array}$ \\
\hline
\end{tabular}

bone and fascia. Compartment syndrome can be acute or chronic. Acute compartment syndrome is a surgical emergency that requires fasciotomy for decompression and preservation of remaining viable tissue. Chronic and exertional compartment syndromes are aggravated by intense activity. On US and MRI, edema and swelling efface the normal muscle striations [22]. Following contrast administration, ischemic muscle enhances heterogeneously, whereas infarcted muscle lacks enhancement. Long-term complications include scarring, atrophic change, and mineralization. Calcific myonecrosis represents a severe complication of post-traumatic compartment syndrome and typically affects the anterior compartment of the lower leg.

\subsection{Myositis Ossificans}

Heterotopic ossification is biologically and histologically identical to normal bone but is located in soft tissues [23]. Many different insults can damage tissue and trigger a physiological reaction that leads to the formation of heterotopic bone. For example, orthopedic surgeries may be complicated by postoperative heterotopic ossification following hardware implantation, arthroplasty, and fracture fixation. Juxtaarticular heterotopic ossification may develop in immobilized patients after severe burn or brain or spinal cord injury. Surgical resection may be necessary to relieve debilitating pain and mechanical symptoms. On radiographs, mature heterotopic ossification demonstrates a pathognomonic cortical shell. On CT, low attenuation of the central cavity follows the attenuation of fat in cancellous bone. On MRI, heterotopic ossification can be misdiagnosed as lipoma because the cortical shell can have the appearance of a fibrous pseudocapsule, and the cancellous bone can demonstrate reticulations similar to adipose tissue [24].

The most frequent type of heterotopic ossification is myositis ossificans (also known as myositis ossificans circumscripta and myositis ossificans traumatica) which usually results from traumatic injury [25]. Myositis ossificans evolves in three general stages [26]. During the first 3-4 weeks, tissue injury causes organizing fibroblastic reaction, osteoblastic differentiation, and osteoid formation. During the second 3-4 weeks, the osteoid matrix becomes mineralized and produces immature lamellar bone. Finally, after 8-10 weeks, immature bone progresses to mature bone with characteristic cortex and intramedullary cavity. The imaging findings evolve with each of the three general stages. Radiographs are negative at first. If the patient presents with a painful, palpable mass, MRI is requested because of the concern for neoplasm. MRI features depend on the degree of tissue damage and surrounding inflammation [27]. MRI can show a mass-like lesion with enhancement following contrast administration simulating sarcoma. If the lesion is biopsied at this early stage, histopathological analysis can lead to the misdiagnosis of pseudo-malignancy [28]. In the middle stages of evolution, radiographs and CT show a zonal arrangement of perimeter mineralization. After full maturation, myositis ossificans exhibits a pathognomonic rind of cortex that is better characterized by radiographs and CT than MRI (Fig. 17.12).

\section{Key Point}

- In the early stage of myonecrosis, MRI can show an enhancing mass-like lesion simulating sarcoma. 

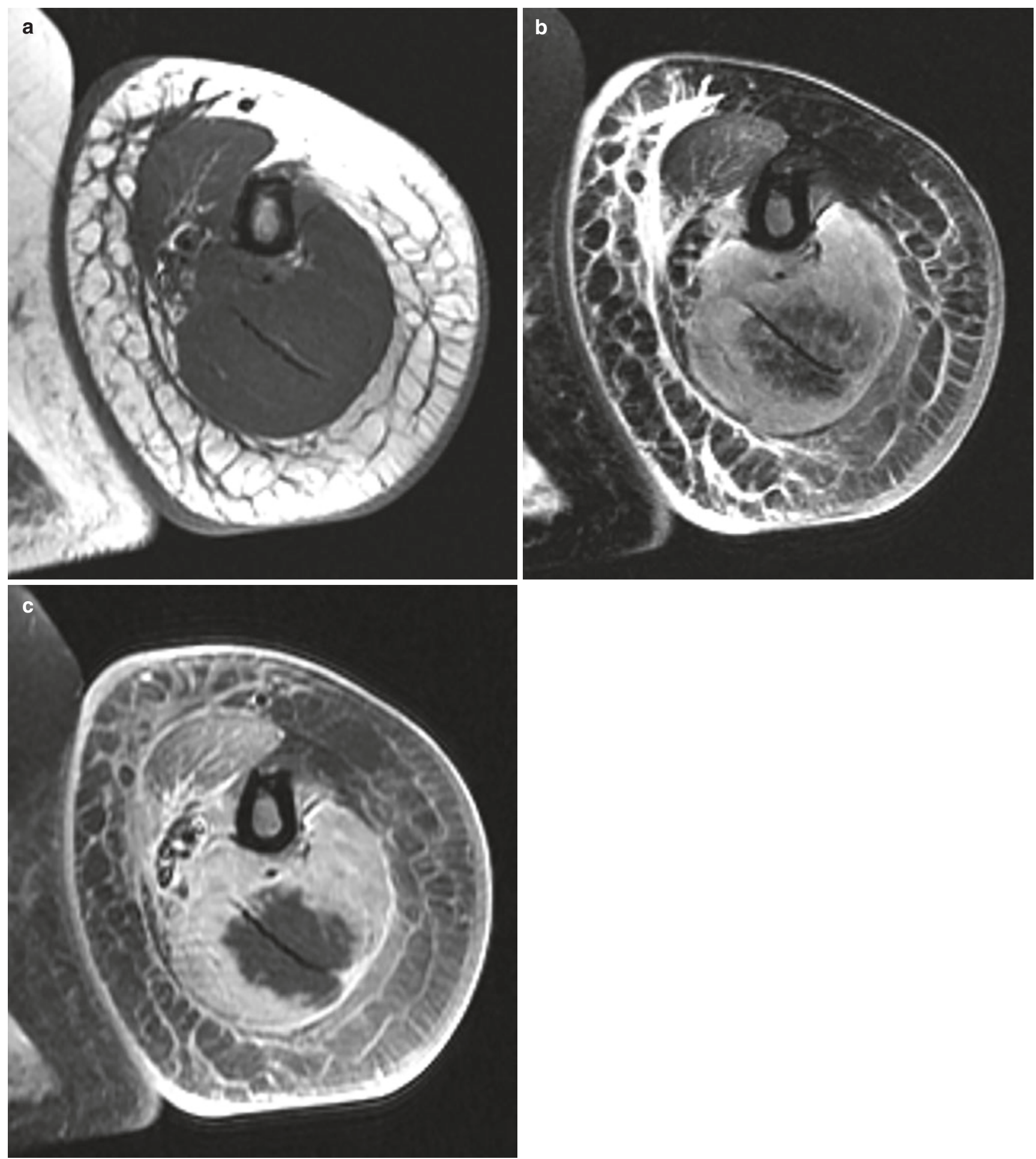

Fig. 17.11 Myonecrosis in a 73-year-old woman complaining of arm pain following drug overdose and stroke. (a) T1-weighted axial image shows swelling of the triceps muscle. (b) T2-weighted, fat-suppressed axial image shows diffuse edema of triceps muscle and low-signal

region surrounding the central tendon. (c) Following intravenous contrast administration, T1-weighted, fat-suppressed axial image shows non-enhancing infarcted muscle corresponding to the low-signal T2-weighted region 


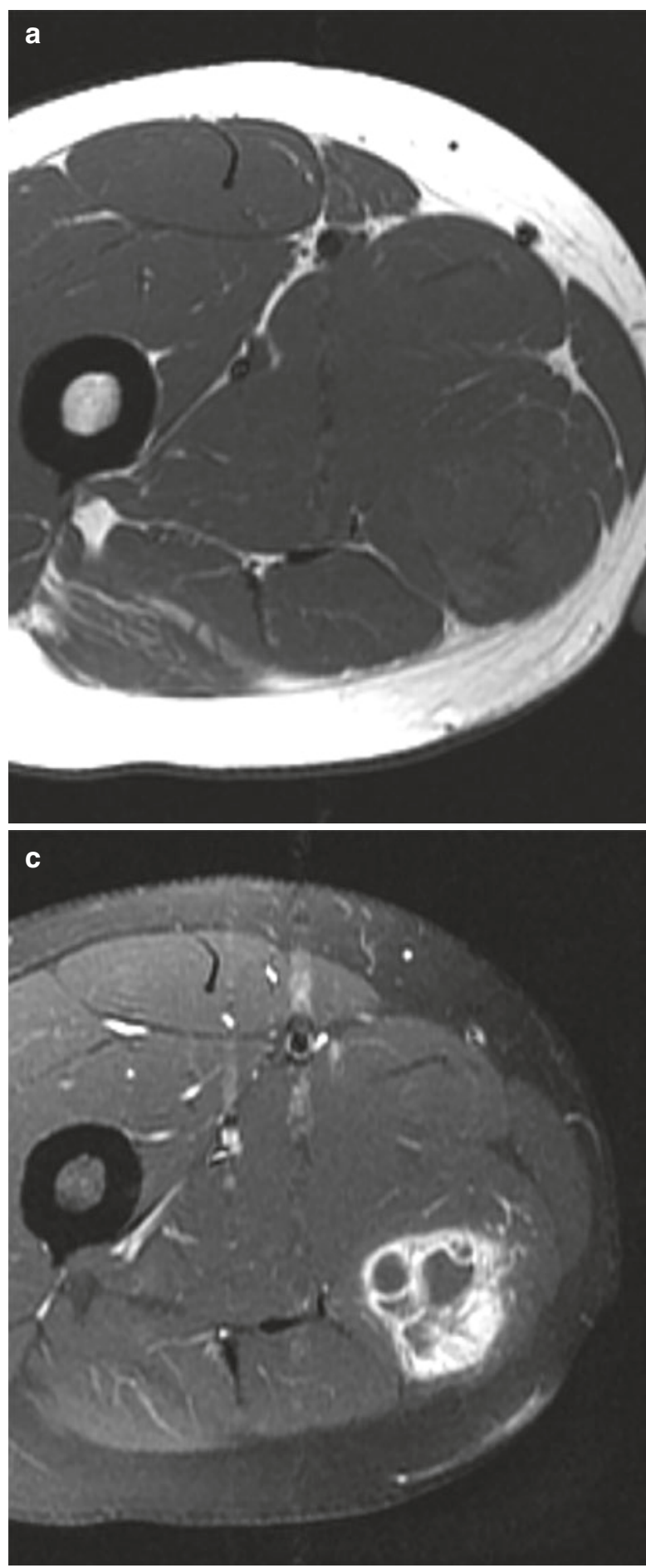

Fig. 17.12 Myositis ossificans in a 31-year-old man presenting with a painful, palpable thigh mass. (a) T1-weighted axial image demonstrates skin marker and swelling of underlying adductor muscle. (b) T2-weighted, fat-suppressed axial image demonstrates a sharply demarcated mass with cystic and solid regions. (c) Following intravenous contrast administration, T1-weighted, fat-suppressed axial image demonstrates dense enhancement of periphery, septations, and solid
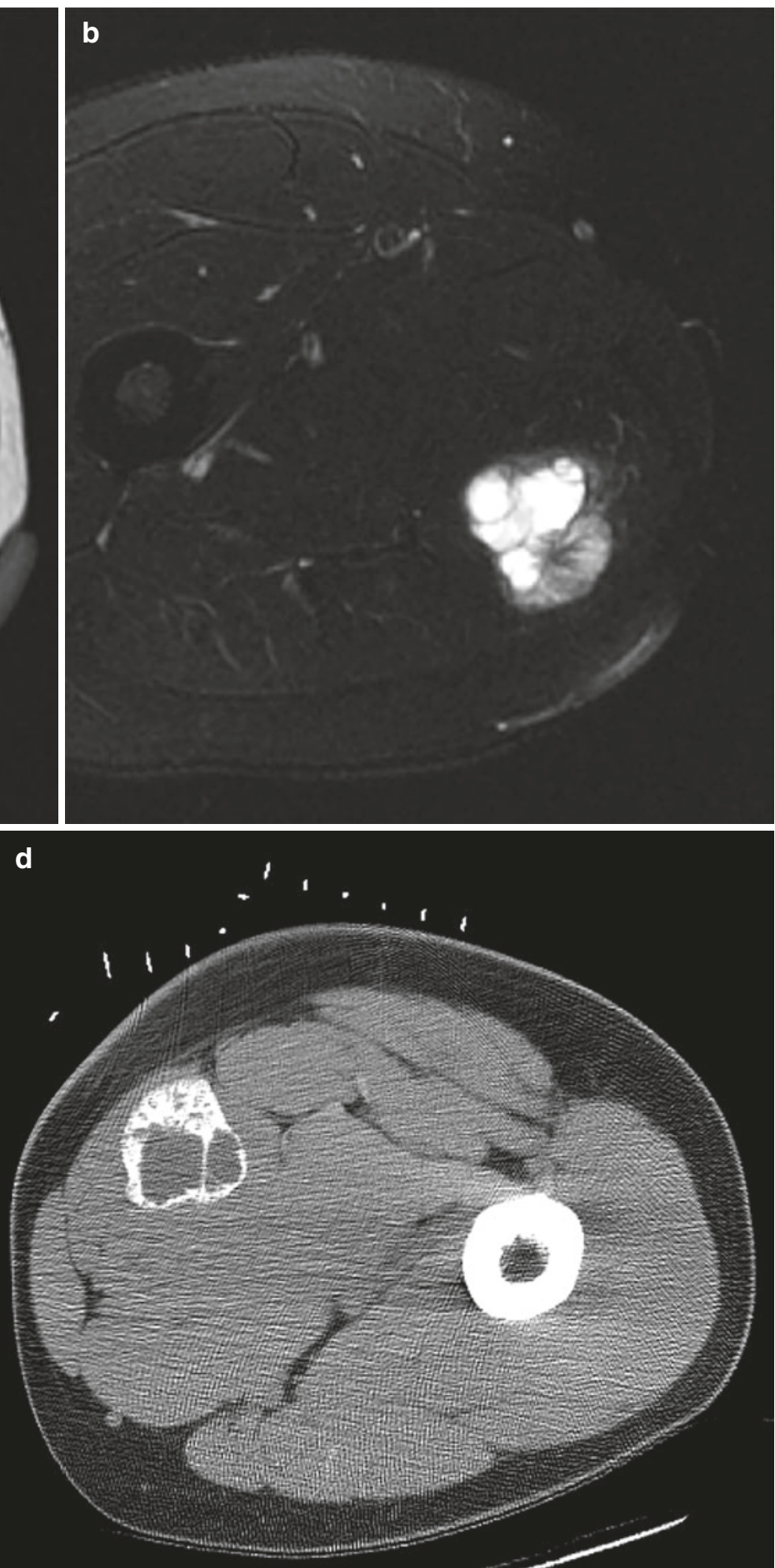

regions. (d) The patient was referred for CT-guided biopsy 2 weeks after the MRI study. With the patient prone, axial image obtained for procedural planning demonstrates grid placement and mineralized lesion corresponding to the MRI abnormality. The biopsy was canceled due to predominantly peripheral mineralization in pattern typical for myositis ossificans 


\subsection{Myopathy}

Myopathy refers to the dysfunction of skeletal muscle and encompasses a spectrum of diseases. Symptoms include myalgias, muscle tenderness, and weakness. Hereditary myopathies result from enzymatic deficiencies and inborn errors of metabolism (e.g., glycogen storage disease). Endocrine myopathies are caused by adrenal, thyroid, parathyroid, and pituitary disorders. Myopathies may be induced by numerous different drugs (e.g., statin, steroid). Myositis is a subcategory of myopathy and refers to the presence of inflammation on histopathological analysis. Inflammatory myopathies are idiopathic, immune-mediated diseases such as dermatomyositis, polymyositis, and inclusion body myositis. They include conditions that affect multiple organs such as sarcoidosis. Myositis can be associated with viral infection (e.g., HIV) or bacterial infection. A complication of bacterial infection is pyomyositis. On MRI, edemalike signal, fatty muscle infiltration, and atrophic change can correlate with disease activity, intensity, and chronicity [19]. On fluid-sensitive MRI sequences, active disease locations show edema-like signal that can provide targets for biopsy [29]. Regions of active inflammation enhance after contrast administration. T1-weighted MRI images are useful for estimating the degree of muscle atrophy and fat replacement related to long-standing or burned-out myositis. Whole-body MRI provides an assessment of disease distribution and helps to gauge therapeutic response. PET can demonstrate corresponding regions of FDG uptake due to hypermetabolism.

\subsection{Necrotizing Fasciitis}

In necrotizing fasciitis, aggressive bacterial infection spreads rapidly between muscle compartments along deep fascial planes and subcutaneous fat. The organisms produce toxins that infarct and liquefy muscle and fat. After a short period of nonspecific symptoms, necrotizing fasciitis swiftly progresses to limb discoloration, systemic toxicity, and sepsis. Although antibiotics can be successful against nonnecrotizing cellulitis and fasciitis, necrotizing infections are often fatal without surgical treatment such as debridement, fasciotomy, or amputation [30]. In patients with suspected necrotizing fasciitis, CT is obtained first because of its availability and sensitivity for gas in the soft tissues. The presence of gas is a specific but insensitive imaging sign. Therefore, the lack of gas cannot exclude necrotizing fasciitis. CT and MRI depict the size and locations of fluid collections [31]. Although intravenous contrast can better delineate muscle liquefaction and tissue infarction, intravenous contrast is contraindicated when necrotizing fasciitis is complicated by rhabdomyolysis and renal failure. Nonspecific imaging features include subcutaneous edema, muscle edema, fascial enhancement, and small intracompartmental fluid collections [32].

\section{Key Point}

- In patients with suspected necrotizing fasciitis, the presence of gas on CT is a specific but insensitive imaging sign. The lack of gas cannot exclude necrotizing fasciitis.

\subsection{Denervation Myopathy}

Denervation occurs when the nerve supply to muscle is partially or completely blocked. This blockade can be symptomatic or asymptomatic and temporary or permanent. More common etiologies include penetrating trauma, surgical transection, and prolonged stretching or compression. Less common etiologies include autoimmune disease, viral infection, and carcinomatosis (nerve infiltration by malignancy). In some locations such as the spinoglenoid notch in the shoulder, slowly growing lesions (e.g., paralabral cyst, intraneural ganglion) may compress the neurovascular bundle and gradually lead to myopathic symptoms. In the acute stages of denervation, MRI shows diffuse muscle edema with uniform contrast enhancement. Muscle bulk remains normal. In the later stages of denervation, MRI findings can overlap with other disorders. Denervated muscle can become atrophic and infiltrated by fat. Edema-like signal may or may not be present. Parsonage-Turner syndrome, also known as idiopathic brachial neuritis, affects one or more muscles of the shoulder girdle. Progressive pain and weakness can mimic the clinical presentation of other shoulder disorders such as rotator cuff tear or capsulitis and, therefore, lead to a delay in diagnosis [33]. In Parsonage-Turner syndrome, supraspinatus and infraspinatus muscles are more commonly involved than deltoid, teres minor, and subscapularis muscles.

\subsection{Muscle Lesions: Differential Considerations}

Myotendinous strain shows pathognomonic localization to the myotendinous junction [34]. Other disorders show a random distribution in muscle. Any localization to the myotendinous junction is coincidental. For example, focal myositis may simulate acute strain if it happens to be contiguous with the myotendinous junction. However, it is usually located in the muscle 
belly separate from the myotendinous junction. Intramuscular neoplasms can displace the myotendinous junction due to mass effect, but they rarely disrupt or encase it.

In myonecrosis, nonviable and adjacent viable muscle can have identical appearances on T1-weighted, T2-weighted, and STIR images because infarcted muscle maintains its normal fiber architecture, similar to necrotic cancellous bone which maintains its normal trabecular architecture. Therefore, the diagnosis of myonecrosis is made with greatest confidence when intravenous contrast is injected. The non-enhancing, avascular tissue stands out prominently against the densely enhancing viable muscle. Although infection (e.g., pyomyositis) can also cause diffuse muscle edema, non-enhancing phlegmon or abscess is distinguished from myonecrosis by the disruption of muscle architecture.

On MRI, hematoma is characterized by a bull's-eye configuration due to degradation of blood products, hemoglobin breakdown, and hemosiderin deposition. On T1-weighted images, the central region is increased in signal intensity. Neoplasms containing fat, such as hemangioma, angiolipoma, and liposarcoma, may also have a central region that is increased in T1-weighted signal intensity and, therefore, similar in appearance to intramuscular hematoma. On fatsuppressed images, however, the signal from fat is decreased, whereas the signal from hematoma is unchanged. Neoplasms complicated by hemorrhagic necrosis also can be mistaken for hematoma, but they have mass affect and displace surrounding structures, including the myotendinous junction. Hemorrhagic necrosis can be mistaken for enhancement following intravenous contrast administration.

Myositis ossificans can pose diagnostic pitfalls. It may present as a painful, palpable soft tissue mass with vague, remote, or absent history of trauma. Clinically, these symptoms and signs are suspicious for neoplasm. On MRI, myositis ossificans has a variable appearance depending on the age of injury, extent of myonecrosis, and degree of adjacent inflammation. In earlier stages, a mass-like abnormality can enhance densely after contrast administration, support the clinical suspicion of malignancy, and result in patient referral to an oncological center for biopsy and surgical resection. Imaging evidence of benign myositis ossificans may not occur until the time of CT-guided biopsy, when the pre-procedural scan demonstrates a characteristic peripheral rim of calcification (Fig. 17.12). In that case, the biopsy can be canceled.

\subsection{Concluding Remarks}

Although muscle is susceptible to multiple traumatic, inflammatory, neoplastic, and neuropathic disorders, imaging often enables a narrow differential diagnosis. Acute traumatic and activity-related injuries such as strain, contusion, and overuse may be complicated by myonecrosis, myositis ossificans, or compartment syndrome. Chronic sequelae are less specific, including muscle atrophy and fatty infiltration. In inflammatory myopathy, imaging is useful clinically in guiding biopsy and assessing treatment response. Imaging has a critical role in the early diagnosis and characterization of necrotizing fasciitis.

\section{Take Home Messages}

- Different imaging modalities have targeted roles in assessing muscle disorders.

- In most muscle disorders, imaging enables a narrow differential diagnosis.

- Clinical and imaging features of early myositis ossificans can simulate sarcoma.

- Contrast administration may be necessary to confirm the diagnosis of myonecrosis.

\section{References}

1. Flores DV, Gómez CM, Estrada-Castrillón M, Smitaman E, Pathria MN. MR imaging of muscle trauma: anatomy, biomechanics, pathophysiology, and imaging appearance. Radiographics. 2017;38(1):124-48.

2. Mueller-Wohlfahrt HW, Haensel L, Mithoefer K, Ekstrand J, English B, McNally S, Orchard J, van Dijk CN, Kerkhoffs GM, Schamasch P, Blottner D, Swaerd L, Goedhart E, Ueblacker P. Terminology and classification of muscle injuries in sport: the Munich consensus statement. Br J Sports Med. 2013;47(6):342-50.

3. Grassi A, Quaglia A, Canata GL, Zaffagnini S. An update on the grading of muscle injuries: a narrative review from clinical to comprehensive systems. Joints. 2016;4(1):39-46. https://doi. org/10.11138/jts/2016.4.1.039.

4. Blankenbaker DG, De Smet AA. MR imaging of muscle injuries. Appl Radiol. 2004;33:14-26.

5. Gyftopoulos S, Rosenberg ZS, Schweitzer ME, et al. Normal anatomy and strains of the deep musculotendinous junction of the proximal rectus femoris: MRI features. AJR Am J Roentgenol. 2008;190:W182-6.

6. Dixon J. Gastrocnemius vs. soleus strain: how to differentiate and deal with calf muscle injuries. Curr Rev Musculoskelet Med. 2009;2:74-7.

7. Longo V, Jacobson JA, Fessell DP, Mautner K. Ultrasound findings of delayed-onset muscle soreness. J Ultrasound Med. 2016;35(11):2517-21.

8. Theodorou DJ, Theodorou SJ, Kakitsubata Y. Skeletal muscle disease: patterns of MRI appearances. $\mathrm{Br} \mathrm{J}$ Radiol. 2012;85(1020):e1298-308.

9. Beggs I. Sonography of muscle hernias. Am J Roentgenol. 2003;180:395-9.

10. Mellado JM, Pérez del Palomar L. Muscle hernias of the lower leg: MRI findings. Skelet Radiol. 1999;28(8):465-9.

11. Adams MA, Chow CK, Premkumar A, Plotz PH. The idiopathic inflammatory myopathies: spectrum of MR imaging findings. RadioGraphics. 1995; 15:563-74.

12. Hernandez RJ, Sullivan DB, Chenevert TL, Keim DR. MR imaging in children with dermatomyositis: musculoskeletal findings 
and correlation with clinical and laboratory findings. AJR Am J Roentgenol. 1993;161(2):359-66.

13. Sanyal S, Atwal SS, Mondal D, Garga UC. Radiographic patterns of soft tissue calcinosis in juvenile dermatomyositis and its clinical implications. J Clin Diagn Res. 2014;8(12):RD08-11.

14. Phillips BA, Cala LA, Thickbroom GW, Melsom A, Zilko PJ, Mastaglia FL. Patterns of muscle involvement in inclusion body myositis: clinical and magnetic resonance imaging study. Muscle Nerve. 2001;24(11):1526-34.

15. Reimers CD, Schedel H, Fleckenstein JL, Nägele M, Witt TN, Pongratz DE, Vogl TJ. Magnetic resonance imaging of skeletal muscles in idiopathic inflammatory myopathies of adults. J Neurol. 1994;241(5):306-14.

16. Mendell JR, Sahenk Z, Gales T, Paul L. Amyloid filaments in inclusion body myositis: novel findings provide insight into nature of filaments. Arch Neurol. 1991;48(12):1229-34.

17. Cox FM, Reijnierse M, van Rijswijk CS, Wintzen AR, Verschuuren JJ, Badrising UA. Magnetic resonance imaging of skeletal muscles in sporadic inclusion body myositis. Rheumatology (Oxford). 2011;50(6):1153-61.

18. Fraser DD, Frank JA, Dalakas M, Miller FW, Hicks JE, Plotz P. Magnetic resonance imaging in the idiopathic inflammatory myopathies. J Rheumatol. 1991;18(11):1693-700.

19. Smitaman E, Flores DV, Mejía Gómez C, Pathria MN. MR imaging of atraumatic muscle disorders. Radiographics. 2018;38(2):50022. https://doi.org/10.1148/rg.2017170112.

20. Filli L, Winklhofer S, Andreisek G, Del Grande F. Imaging of myopathies. Radiol Clin N Am. 2017;55(5):1055-70. https://doi. org/10.1016/j.rcl.2017.04.010.

21. Cunningham J, Sharma R, Kirzner A, et al. Acute myonecrosis on MRI: etiologies in an oncological cohort and assessment of interobserver variability. Skelet Radiol. 2016;45(8):1069-78. https://doi. org/10.1007/s00256-016-2389-4.

22. Rominger MB, Lukosch CJ, Bachmann GF. MR imaging of compartment syndrome of the lower leg: a case control study. Eur Radiol. 2004;14(8):1432-9.
23. McCarthy EF, Sundaram M. Heterotopic ossification: a review. Skelet Radiol. 2005;34:609-19.

24. Palmer WE, Bancroft L, Bonar F, et al. Glossary of terms for musculoskeletal radiology. Skeletal Radiol. 2020;49:1-33. https://doi. org/10.1007/s00256-020-03465-1.

25. Robinson P, Salehi F, Grainger A, et al. Cadaveric and MRI study of the musculotendinous contributions to the capsule of the symphysis pubis. AJR Am J Roentgenol. 2007;188(5):W440-5.

26. Kransdorf MJ, Meis JM, Jelinek JS. Myositis ossificans: MR appearance with radiologic-pathologic correlation. AJR Am J Roentgenol. 1991;157(6):1243-8.

27. De Smet AA, Norris MA, Fisher DR. Magnetic resonance imaging of myositis ossificans: analysis of seven cases. Skelet Radiol. 1992;21:503-7.

28. Nuovo MA, Norman A, Chumas J, Ackerman LV. Myositis ossificans with atypical clinical, radiographic, or pathologic findings: a review of 23 cases. Skelet Radiol. 1992;21:87-101.

29. Endo Y, Miller TT. Myositis and fasciitis: role of imaging. Semin Musculoskelet Radiol. 2018;22(3):286-98.

30. Edlich RF, Cross CL, Dahlstrom JJ, Long WB. Modern concepts of the diagnosis and treatment of necrotizing fasciitis. J Emerg Med. 2010;39(2):261-5. https://doi.org/10.1016/j. jemermed.2008.06.024

31. Kim KT, Kim YJ, Won Lee J, et al. Can necrotizing infectious fasciitis be differentiated from nonnecrotizing infectious fasciitis with MR imaging? Radiology. 2011;259(3):816-24. https://doi. org/10.1148/radiol.11101164.

32. Schmid MR, Kossmann T, Duewell S. Differentiation of necrotizing fasciitis and cellulitis using MR imaging. AJR Am J Roentgenol. 1998;170(3):615-20.

33. Parsonage MJ, Turner JW. Neuralgic amyotrophy; the shouldergirdle syndrome. Lancet. 1948;1(6513):973-8.

34. Palmer WE, Kuong SJ, Elmadbouh HM. MR imaging of myotendinous strain. AJR Am J Roentgenol. 1999;173(3):703-9.

Open Access This chapter is licensed under the terms of the Creative Commons Attribution 4.0 International License (http://creativecommons. org/licenses/by/4.0/), which permits use, sharing, adaptation, distribution and reproduction in any medium or format, as long as you give appropriate credit to the original author(s) and the source, provide a link to the Creative Commons license and indicate if changes were made.

The images or other third party material in this chapter are included in the chapter's Creative Commons license, unless indicated otherwise in a credit line to the material. If material is not included in the chapter's Creative Commons license and your intended use is not permitted by statutory regulation or exceeds the permitted use, you will need to obtain permission directly from the copyright holder. 